\title{
Study Registry Acronym
}

National Cancer Institute

\section{Source}

National Cancer Institute. Study Registry Acronym. NCI Thesaurus. Code C94135.

The non-unique initials or abbreviated name used for identification of the registry. 